تناسث توزيع الماء في المنطقة الجذرية

\author{
حقي إسماعيل ياسين \\ أستاذ مساعد/كلية الهنسسة/جامعة الموصل
}

\begin{abstract}
المستخلص
إن أداء منظومة الري يتم تقييمه بمعايير توضح استغلال مياه الري في الحقل وهي كفاءة الإرواءو وكفاية الإرواء

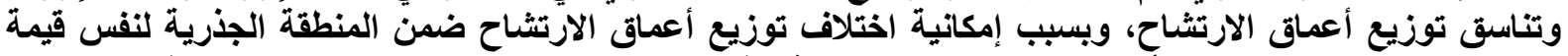

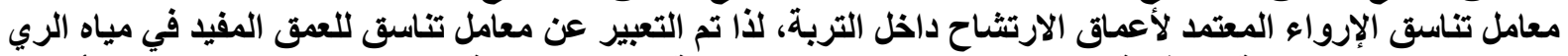

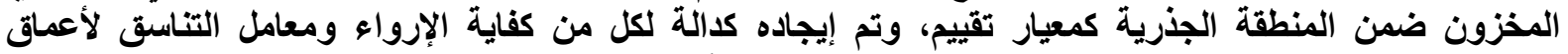
الارتثاح وذلك لكل من حالتي التوزيع الخطي والتئية ونيع الطبيعي لأعماق الارتثاح. الكلمات الدالة: توزيع أعماق الارتثاح، تناسق الإرواء، كفاية الإرواء، كفاءة الإرواء.
\end{abstract}

\title{
Water Distribution Uniformity In The root Zone
}

\section{Haqqi I. Yasin}

\begin{abstract}
The performance of irrigation systems can be evaluated by criteria which show the efficient use of on-farm water distribution. The different distribution of infiltration depths in the soil may give the same value of application uniformity coefficient; therefore the present study considered the distribution uniformity coefficient of the useful infiltration depths in the root zone as part of the evaluation criteria. The considered criteria was expressed as a function of both application adequacy and the application uniformity of infiltration depths. The derived function is applicable for linear and natural distribution of infiltration depths.
\end{abstract}

Key words: infiltration depths distribution, application uniformity, application adequacy, application uniformity 


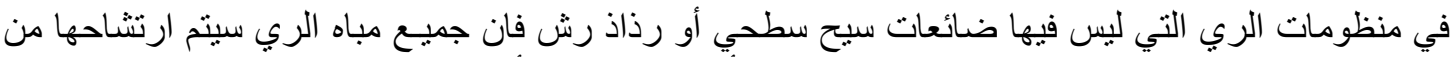

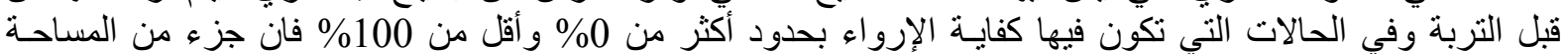

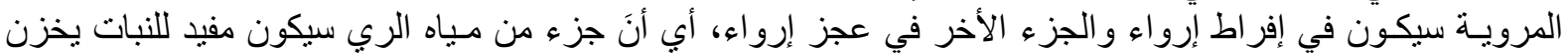

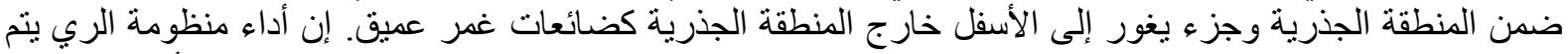

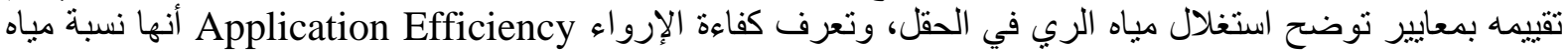

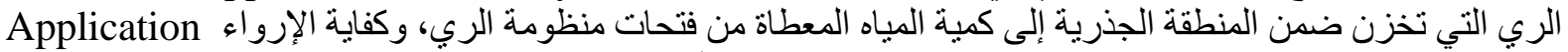

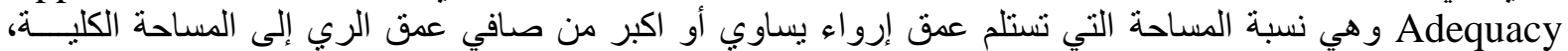
وتناسق الإرو اء Application Uniformity و هو ما يعبر عن انتظام توزيع مياه الري.

وقد أوضحت معظم الدراسات إن أوفق توزيع لأعماق الارتشاح مع المساحة هما التوزيع الخطي (Linear)

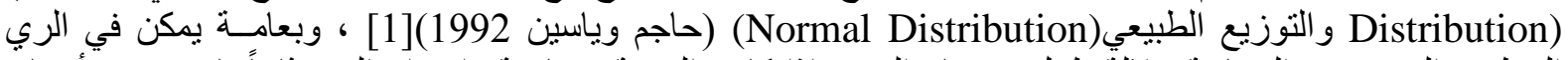

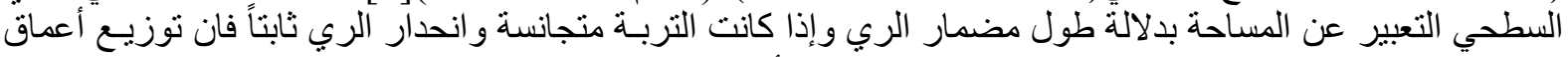

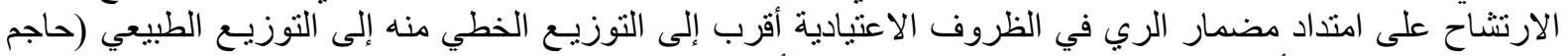

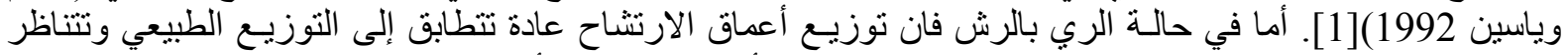

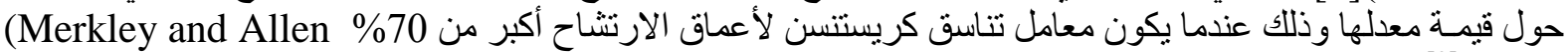

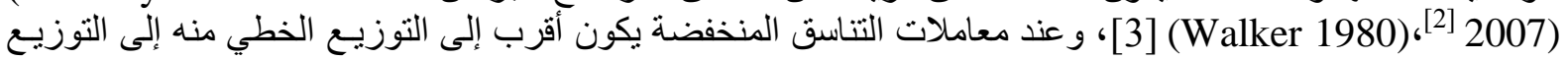

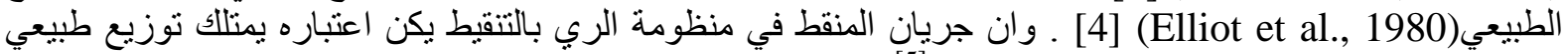

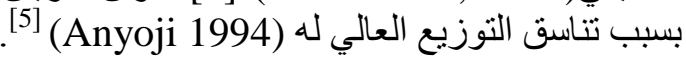

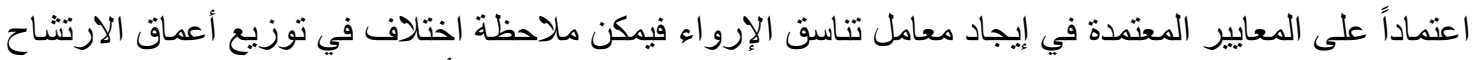

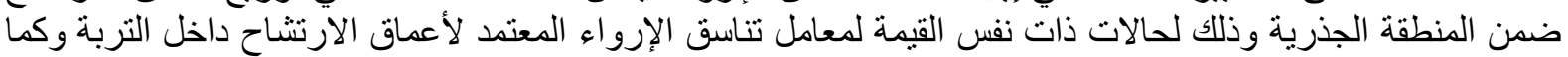

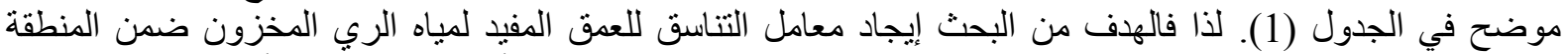

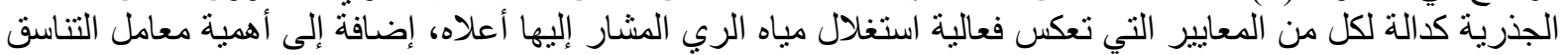

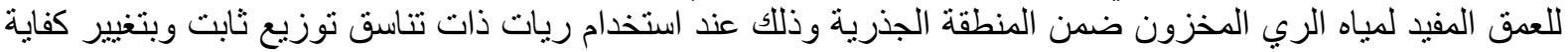
الارواء من خلال التحكم بفاصلة الإرواء.

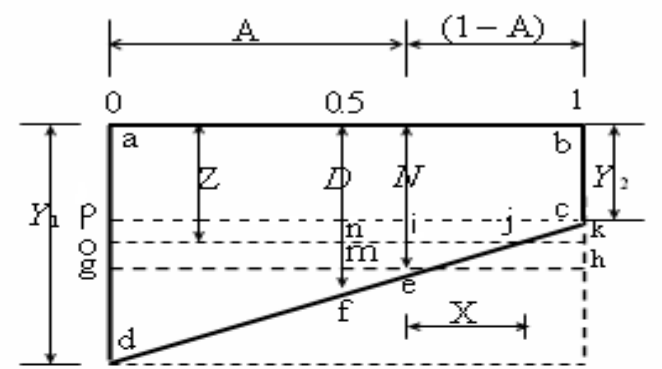

التوزيع الخطي (Linear Distribution)

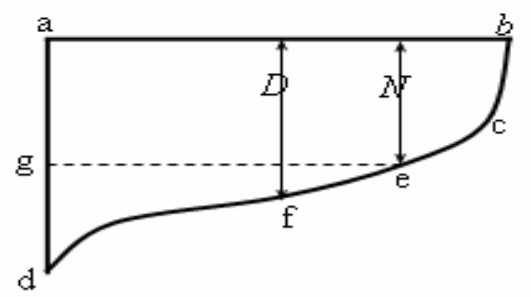

التوزيع الطنبيعي(Normal Distribution)

\section{توزيع أعماق الارتثاح}

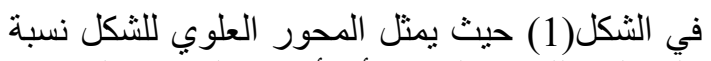

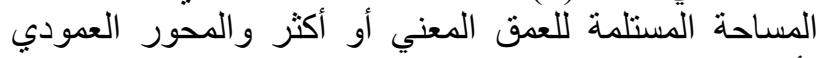

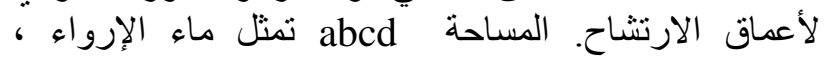
و المساحة abceg تمثل الماء المفيد ضمن المنطقة المباحة الجذرية،

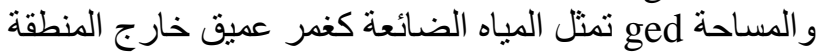

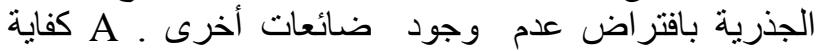

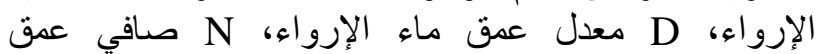

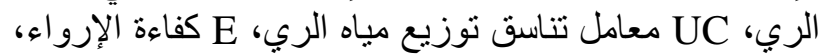
UCU ضمن المنطقة الجذرية.

الثكل(1):توزيع أعماق الارتشاح 
الجدول(1):أربعة مجاميع لأعماق الارتثاح باعتماد صافي عمق الري يساوي معدل الإرواء.

\begin{tabular}{|c|c|c|c|c|c|c|c|c|c|c|c|c|c|c|}
\hline اللتعنقاسق & كالإرواءة & كالإرواءة & تلناسق & & & & & & & & & & & \\
\hline 0.882 & 0.90 & 0.50 & $\mathbf{0 . 8 0}$ & 20 & 22 & 24 & 26 & 28 & 32 & 34 & 36 & 38 & 40 & الأجمولى \\
\hline 0.867 & $\mathbf{0 . 9 0}$ & $\mathbf{0 . 5 0}$ & $\mathbf{0 . 8 0}$ & 16 & 20 & 28 & 28 & 28 & 30 & 34 & 38 & 38 & 40 & الثجانية \\
\hline 0.844 & $\mathbf{0 . 9 0}$ & 0.50 & $\mathbf{0 . 8 0}$ & 15 & 18 & 29 & 29 & 29 & 30 & 33 & 36 & 39 & 42 & الثجاثة \\
\hline $\mathbf{0 . 8 3 0}$ & 0.90 & 0.50 & $\overline{0.80}$ & 04 & 29 & 29 & 29 & 29 & 36 & 36 & 36 & 36 & 36 & المجموعة \\
\hline
\end{tabular}

التوزيع الخطي لأعماق الارتثاح:

للتنوزيع الخطي يمكن التعبير عن معامل التناسق لأعماق الارتثاح بالصيغة الآتية (حاجم وياسين 1992) ${ }^{[6]}$ (Karmeli 1978) ‘[1]

$U C=1-0.25\left(Y_{1}-Y_{2}\right) / D$

حيث Y و وما العمق الأقصى و الأدنى لأعماق الارتشاح بالتوزيع الخطي، ومن تثابه المثلثين dci و emf فانَ: $\left(Y_{1}-Y_{2}\right)=(D-N) /(A-0.5)$

وأنَ معدل العمق المفيد ضمن المنطقة الجذرية يكون الآتي:

$Z=N^{*} A+\left(N+Y_{2}\right)(1-A) / 2$

$(N-Z) / X=\left(N-Y_{2}\right) /(1-A)$ ومن تثابه المثلثين eli و ele فان:

$X=0.5(1-A)^{2}$

وبالتعويض عن قيمة Z من المعادلة 3 في المعادلة 4 ينتج:

وباعتماد نفس المبدأ في التعبير عن معامل التناسق في المعادلة 1 فانً معامل تناسق توزيع العمق المفيد لمياه الري المخزون ضمن المنطقة الجذرية يكون:

$$
U C U=\left\{(N-Z) A+0.5(N-Z) X+0.5\left(Z-Y_{2}\right)(1-A-X)\right\} /(Z * 1)
$$

وبالتعويض عن قيمة Z من العادلة 3 و من المعلة 5 في المعادلة 6 ينتج:

$$
U C U=1-\left\{0.5\left(N-Y_{2}\right)(1-A)(1+A)^{2}\right\} /\left(N * A+N+Y_{2}-A^{*} Y_{2}\right)
$$

ومن تثابه المثلثات ehe و g g و و و و و

$$
\begin{aligned}
& \frac{D-N}{A-0.5}=\frac{N-Y_{2}}{1-A}=\frac{Y_{1}-Y_{2}}{1}=4 D(1-U C) \\
& Y_{2}=N-4 D(1-U C)(1-A) \\
& \left(N-Y_{2}\right)=4 D(1-U C)(1-A)
\end{aligned}
$$

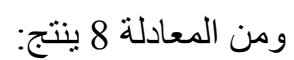




$$
\begin{aligned}
& N=D-4 D(1-U C)(A-0.5) \\
& U C U=\frac{2 D(1-U C)\left(1-A^{2}\right)^{2}}{2 N-4 D(1-U C)\left(1-A^{2}\right)^{2}} \\
& \text { وبالتعويض عن قيمة } Y_{2} \text { من المعادلة } 9 \text { وقيمة (N- } 10 \text { مي } 10 \text { من المعادلة } \\
& \text { في المعادلة } 7 \text { ينتج: } \\
& U C U=1-\frac{(1-U C)\left(1-A^{2}\right)^{2}}{1-2 A^{2}(1-U C)} \quad 12 \text { المعادلة } 11 \text { في المعاديمة فئلة }
\end{aligned}
$$

\section{التوزيع الطبيعي لأعماق الارتثـاح:}

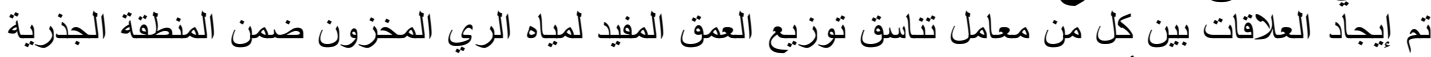

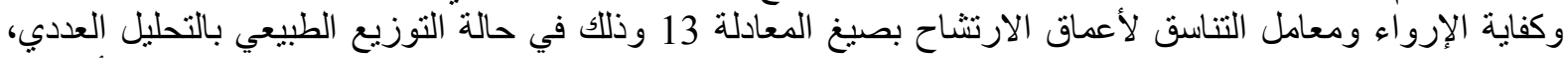

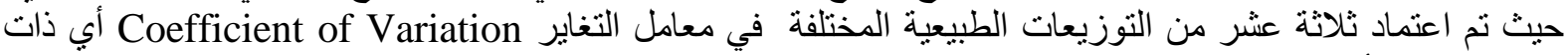

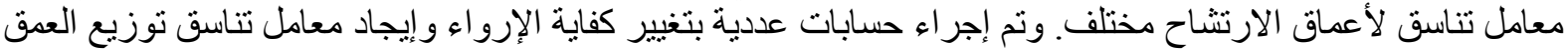

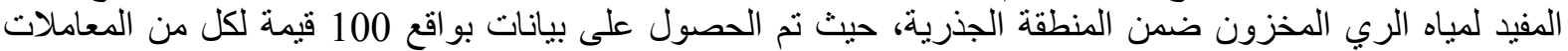

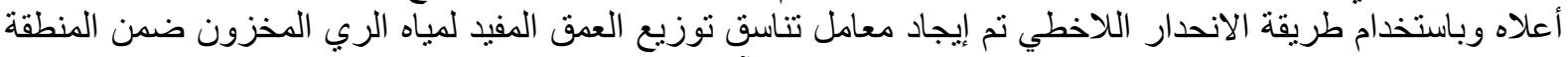
الجذرية كدالة لكل من كفاية ومعامل تناسق الإرواء وباء وبالصيغة الآتية:

$$
U C U=1-\frac{(1-U C)\left(1-A^{2}\right)^{2}}{1-2.1409 A^{2}(1-U C)}
$$

\begin{tabular}{|c|c|c|c|}
\hline المعيد المحسوبة بالمعادئة & العقى المفيد المحسوبية & ومعامل التناسق لأعماق & كفاية الإرواء \\
\hline 0.856 & 0.855 & 0.771 & 0.508 \\
\hline 0.939 & 0.937 & 0.886 & 0.541 \\
\hline 0.983 & 0.983 & 0.962 & 0.590 \\
\hline 0.854 & 0.854 & 0.695 & 0.639 \\
\hline 0.949 & 0.948 & 0.809 & 0.738 \\
\hline 0.988 & 0.987 & 0.924 & 0.787 \\
\hline 0.734 & 0.735 & 0.619 & 0.508 \\
\hline 0.680 & 0.683 & 0.543 & 0.541 \\
\hline 0.855 & 0.855 & 0.657 & 0.689 \\
\hline 0.791 & $\mathbf{0 . 8 0 7}$ & 0.505 & $\mathbf{0 . 7 8 7}$ \\
\hline 0.969 & 0.970 & 0.581 & 0.934 \\
\hline 0.984 & 0.983 & 0.657 & $\mathbf{0 . 9 3 4}$ \\
\hline
\end{tabular}

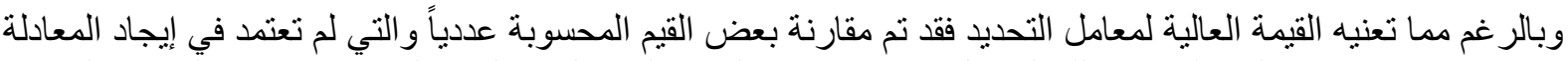

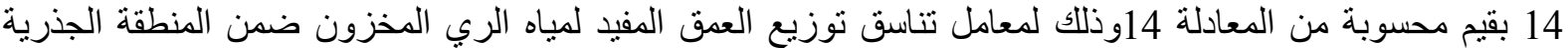

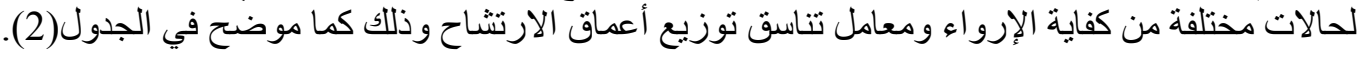

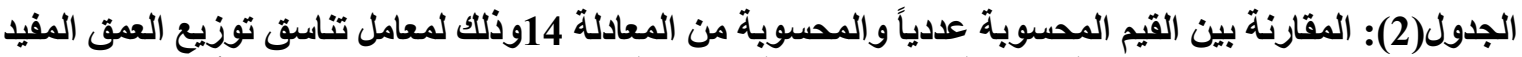

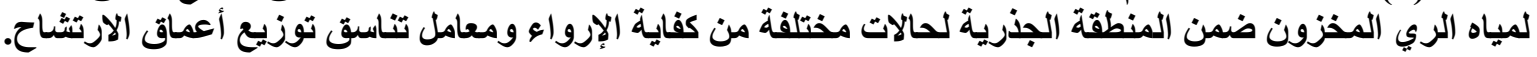




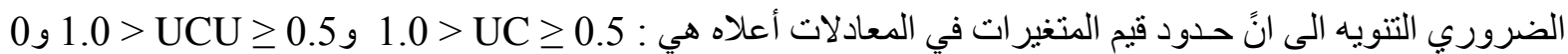
$1.0 \geq \mathrm{E}>0$ و $1.0>\mathrm{A} \geq$ الخلاصة والاســتنتاج

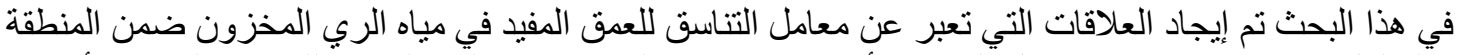

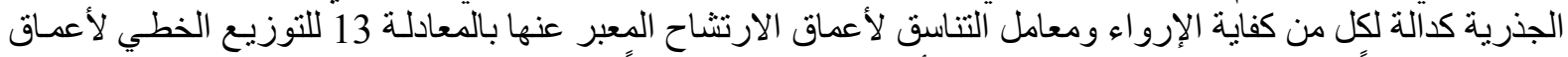

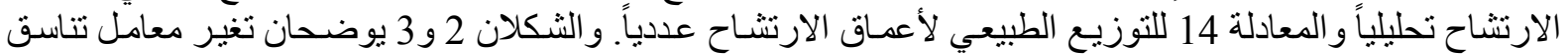

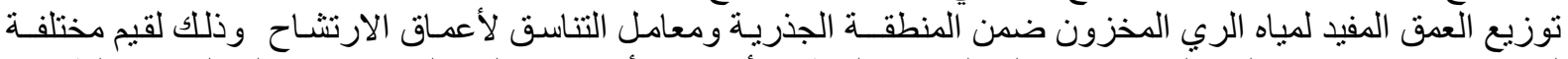

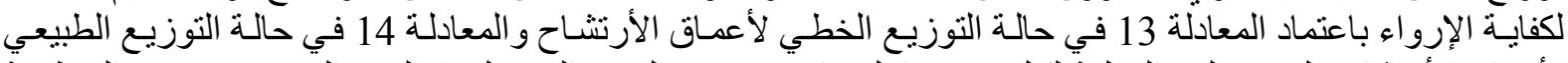

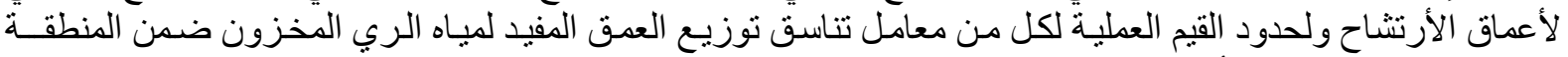

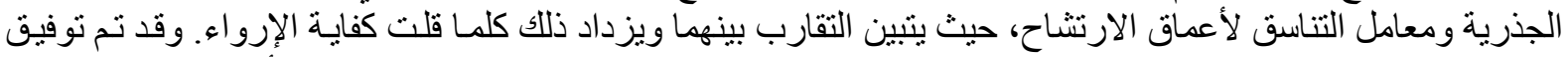

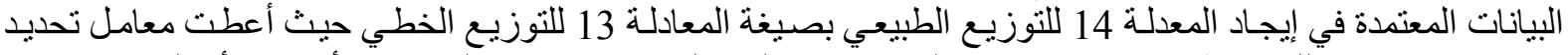

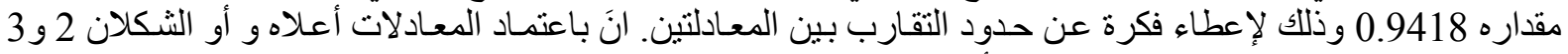

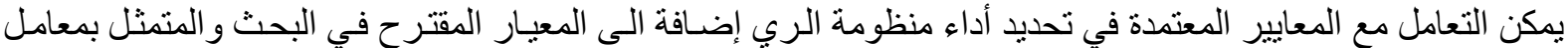

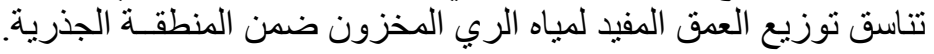

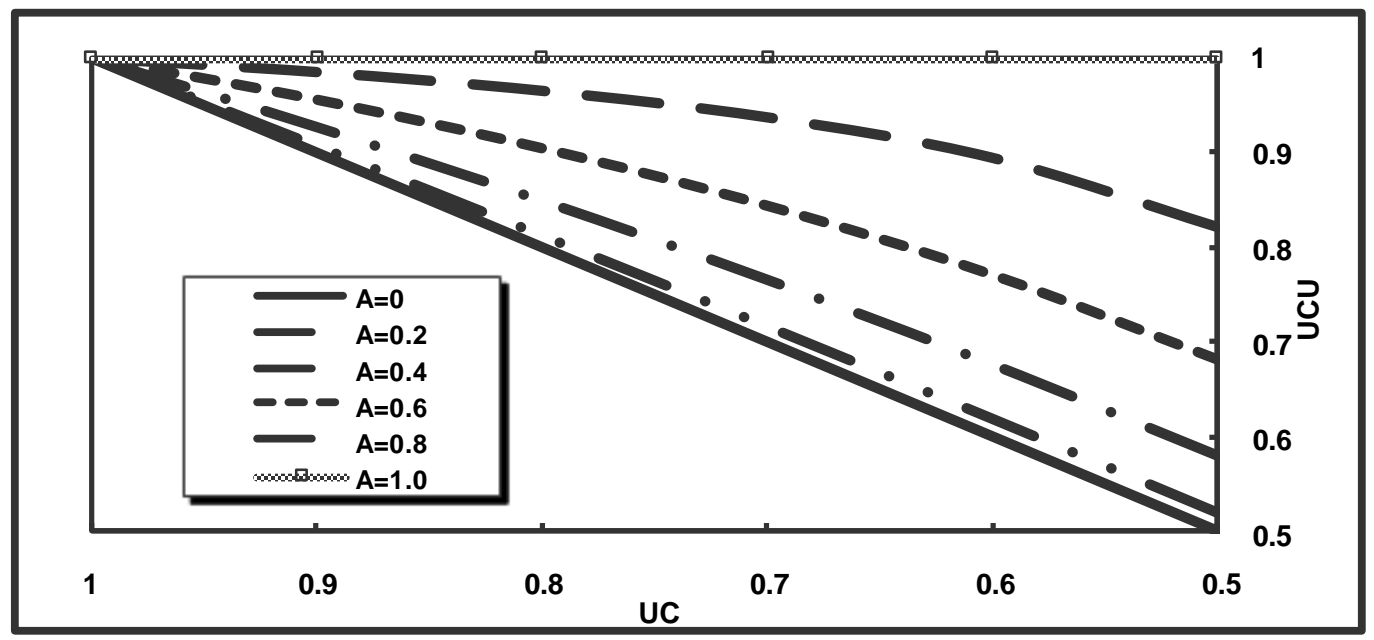

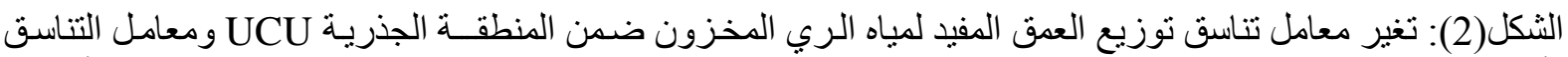

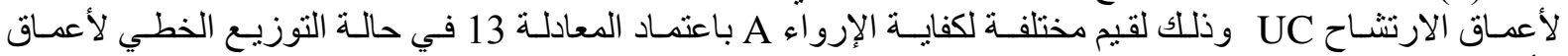
الأرنشاح.

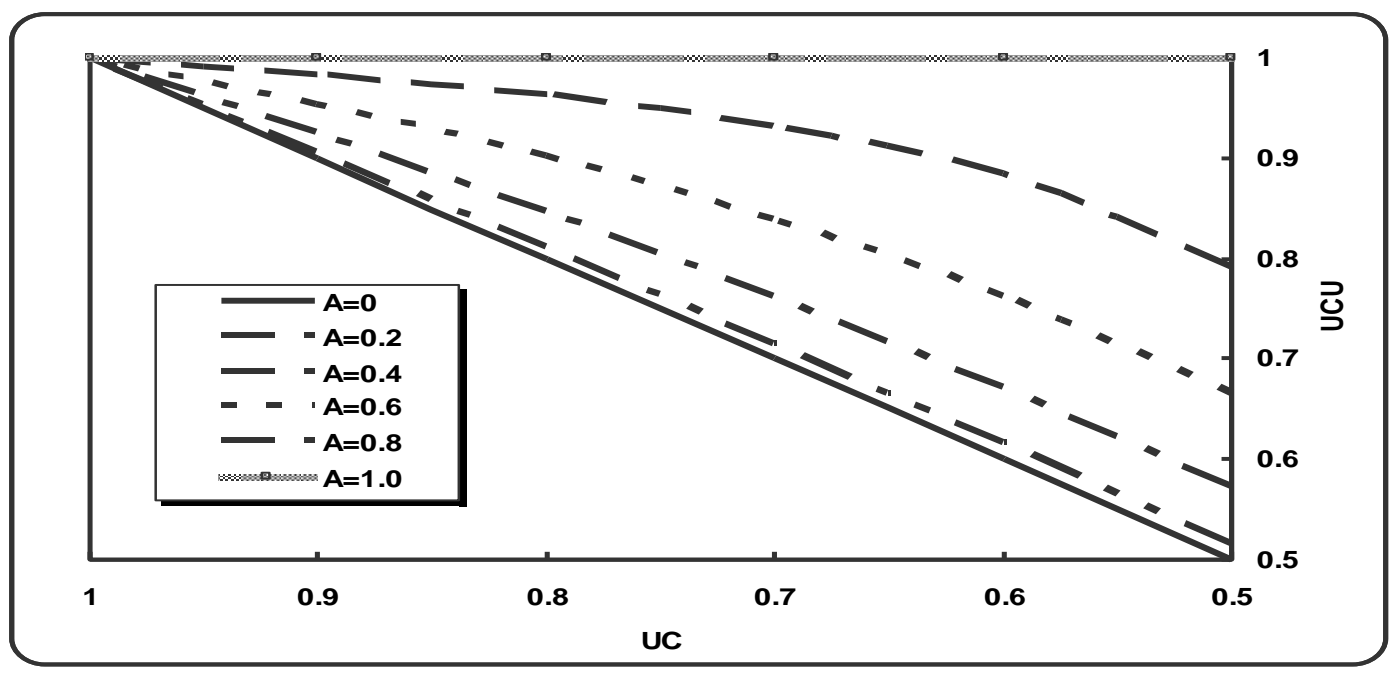




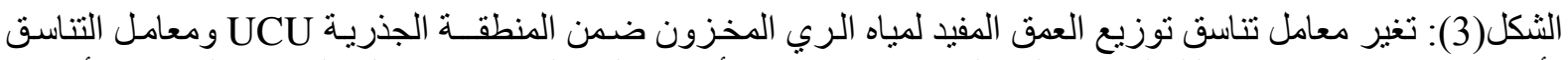

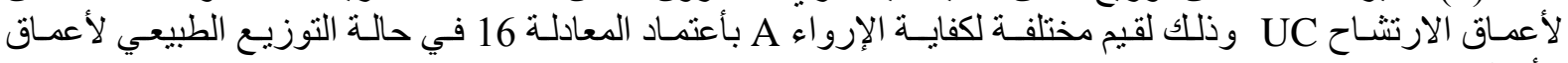
الأرتشاح. المصادر

1. حاجم ، أحمد يوسف و ياسين ,حقي إسماعيل ، هندسة نظم الري الحقلي ، دار الكتب للطباعة والنشر ، جامعة الموصل ، 1992.

2. Merkley G. P and R.G. Allen. 2004. Sprinkler and trickle irrigation lectures. cd3wd.com/CD3WD_40/OCW/IRRIG_SPR_TRICK/ecfile200501048603507665/...

3. Walker W.R. 1980.Sprinkler and trickle irrigation. Colorado State University, Engineering Renewal and Growth, Fort Collins, Colorado 80523.

4. Elliot R.L., J.D. Nelson, J.C. Loftis, and W.E. Hart. 1980.Comparison of sprinkler uniformity models. Journal of Irrigation and Drainage Division, ASCE 106(IR4):321-330.

5. Anyoji H. and I.P. Wu. 1994. Normal distribution water application for drip irrigation schedules. Transactions of the ASAE 37(1):159-164.

6. Karmeli D. 1978. Estimating sprinkler distribution patterns using linear regression. Transactions of the ASAE 21(4):682-686. 